\title{
Covid-19 Vaccination Maternal Services for Pregnant Women in the Work Area of Telaga Community Health Centers
}

\author{
Harismayanti ${ }^{1}$, Rona Febriyona ${ }^{1}$, St. Surya Indah Nurdin ${ }^{1}$, Mukmin Wadila Rahim ${ }^{1}$, Delanti \\ Mahmud $^{1}$, Nurul Hasanah ${ }^{1}$ \\ ${ }^{1}$ Muhammadiyah University of Gorontalo, Indonesia
}

Received: November 8, 2021

Received in Revised: December 15,

Accepted: December 20, 2021

2021

\begin{abstract}
This study aims to provide an overview of how the covid-19 vaccination service for pregnant women in the work area of the Telaga Health Center. This study uses a qualitative method that focuses on ANC services with in-depth interview techniques, data analysis is carried out descriptively and presented in narrative form. This research was carried out in the working area of the Telaga Health Center from September 2021 to November 2022. The informants in this study were pregnant women in the Telaga Health Center working area as many as 7 participants and 2 key informants were the program coordinator midwives. With a snowball sampling technique. The results of the study found that there were 5 themes that described Covid-19 vaccination services for pregnant women, namely Socialization of Covid-19 Vaccination for pregnant women, Issue of KIPI for Covid-19 Vaccination, Supporting Factors for Successful Vaccination in Pregnant Women, Body Response of Pregnant Women after Covid-19 Vaccination. 19, and Covid-19 Vaccination Requirements for Pregnant Women. The mandatory output in this research is the publication of research results in one of the international journals.
\end{abstract}

Keywords: Vaccinations, Covid-19, Pregnant Women, Supporting Factors, AEFI Issue

\section{Introduction}

Neonatal mortality in Indonesia is a problem and challenge that needs to be handled quickly, coupled with the Covid-19 disaster situation, making this problem need special attention from the Government. Maximum maternal service is one of the efforts that need to be done in preventing problems in childbirth (Hanson et al., 2017). The forms of maternal services referred to include services for pregnant women, childbirth, postpartum mothers and newborns (Kerber et al., 2007; Steen et al., 2012). In this Covid-19 pandemic situation, almost all forms of public services, including health services, are subject to restrictions both in terms of the form of service and the number of patient services every day (Sulkowski \& Ignatowski, 2020). Restrictions on health services for pregnant women and newborns are enforced in all health care facilities so that of course the maternal services obtained are very minimal which of course can affect the welfare of mothers and newborns, lack of knowledge of mothers.

Maternal and newborn health services are one form of service that is affected both in terms of access and quality of examinations, such as pregnant women being reluctant to go to the Puskesmas or other health care facilities for fear of being infected, the recommendation to postpone pregnancy checks and classes for pregnant women and the unpreparedness of services from in terms of personnel and infrastructure including Personal Protective Equipment. The government must be able to provide maternal health services for mothers and newborns that are safe, quality and affordable in a fair and equitable manner regardless of differences in 
religion, race/ethnicity, class and social status of individuals, families and communities and become a challenge again during the COVID-19 pandemic.

Based on data from the Task Force for the Acceleration of Handling Covid-19 in September 2020 , the number of confirmed positive Covid-19 patients was $4.9 \%$ or 19 of 1,483 . This data shows that pregnant women in labor, postpartum, and newborns are also vulnerable targets for COVID-19 infection and this condition is feared to increase maternal and newborn morbidity and mortality. One of the efforts that can be made to reduce the mortality rate for pregnant, maternity and postpartum women with COVID-19 is the COVID-19 vaccination for pregnant women (Blakeway et al., 2021).

Vaccination aims to provide immunity against a certain disease so that if one day you are exposed to the disease, you will not get sick or only experience mild illness. With this vaccination, it will provide immunity to fight the incoming virus and vice versa if not vaccinated and when the body's immune system is low, it will be more susceptible to disease or infection. The condition of pregnant women who have a low immune system, covid-19 can infect at any time, especially if the pregnant woman has a history of congenital diseases such as lung disease, asthma, liver damage, heart disease, it will have more severe symptoms.

Corona virus that is infected in pregnant women who have comorbidities will cause more severe symptom effects so that they are at risk of preterm labor, miscarriage, and even death. In accordance with the circular on COVID-19 vaccination for pregnant women issued by the Indonesian Ministry of Health No. HK.02.02/I/2007/2021 that through the screening stage the implementation of vaccines for pregnant women can be carried out using three types of vaccines that are allowed, namely, the Pfizer and Moderna mRNA-covid-19 vaccines and the Sinovac inactivated virus platform vaccine which is adjusted to the availability of the vaccine.

The dose of the first vaccine is started in the second trimester of pregnancy and the second dose is given according to the interval of the type of vaccine (Bookstein Peretz et al., 2021). However, in reality, in the field of many opinions, hoax news circulating related to the effect of vaccination causes pregnant women to be reluctant to vaccinate against covid-19 and also obtained data from the Telaga Health Center until now no one has vaccinated pregnant women because of course the implementation of this vaccination must be accompanied by a letter. Consent of the husband and there is no coercion. This study aims to determine the form of implementation of maternal services for COVID-19 vaccination for pregnant women

\section{Methods}

This research was carried out for a year starting in September 2021-November 2021 which included the stages of proposal, preparation, research process, data processing, and report preparation. While the location of the research was carried out in the Work Area of the Telaga Health Center, Gorontalo Regency. This study uses a qualitative research design with a phenomenological approach that focuses on Covid-19 vaccination services for pregnant women. Data collection techniques with in-depth interviews. Data analysis was carried out descriptively and presented in narrative form. Informants in this study were pregnant women who were in the area of the Telaga Biru Health Center. Research informants were determined by snowball sampling technique with a total of 7 participants. The key informants in this study were 2 program coordinator midwives. 


\section{Results and Discussion}

\section{Characteristics of Participants}

Table 1. Participant Demographic Data

\begin{tabular}{|c|c|c|c|c|c|}
\hline Participant Code & Age & $\begin{array}{c}\text { Gestational } \\
\text { Age }\end{array}$ & Education Final & Job & $\begin{array}{c}\text { Vaccination } \\
\text { Status }\end{array}$ \\
\hline P1 & 30 & 20 & D1 & entrepreneur & \\
\hline P2 & 26 & 23 & junior high school & housewife & Vaccinated \\
\hline P3 & 30 & 30 & senior High School & housewife & Vaccinated \\
\hline P4 & 27 & 31 & senior High School & housewife & Not Vaccine yet \\
\hline P5 & 27 & 24 & D3 & entrepreneur & Vaccinated \\
\hline P6 & 33 & 30 & senior High School & housewife & Not Vaccine yet \\
\hline P7 & 28 & 10 & senior High School & entrepreneur & Not Vaccine yet \\
\hline P8(Key Informant) & 38 & - & D4 & Midwife Coord & Vaccinated \\
\hline P9 (Key Informant) & 40 & - & D4 & Midwife Coord & Vaccinated \\
\hline
\end{tabular}

The number of participants in this study involved 9 participants consisting of 7 pregnant women in the Telaga PKM work area and 2 key informants as coordinator midwives at Telaga PKM. The average age of the participants was between $26-28$ years. Participants' gestational age was between 16 weeks to 31 weeks. The education of participants is quite varied from IRT, self-employed, and health workers. Meanwhile, the vaccination status of pregnant women in all participants has not been vaccinated except for health workers, midwives as key informants.

\section{Thematic Analysis}

This section describes in detail and detail the various themes identified from the data collection results. After going through the data analysis process using content analysis developed by Colazzi. In this study, 5 themes were identified that describe maternal services for COVID-19 vaccination for pregnant women in the Telaga Health Center Work Area, namely Socialization of Covid-19 Vaccination for pregnant women, Issue of KIPI for Covid-19 Vaccination, Supporting Factors for Successful Vaccination in Pregnant Women, Body Response Pregnant Women After Covid-19 Vaccination, and Conditions for Covid-19 Vaccination for Pregnant Women.

\section{Theme I: Socialization of Covid-19 Vaccination for pregnant women}

The socialization of COVID-19 vaccination for pregnant women is one of the factors that hinders maternal services for COVID-19 vaccination (Gupta \& Pushkala, 2021; Alhomaizi et al., 2021). To explore this, the researcher asked: "How do you know about the socialization of maternal care programs for COVID-19 vaccination for pregnant women?" and obtained keywords and categories to describe the theme as shown in Figure 1 below: 


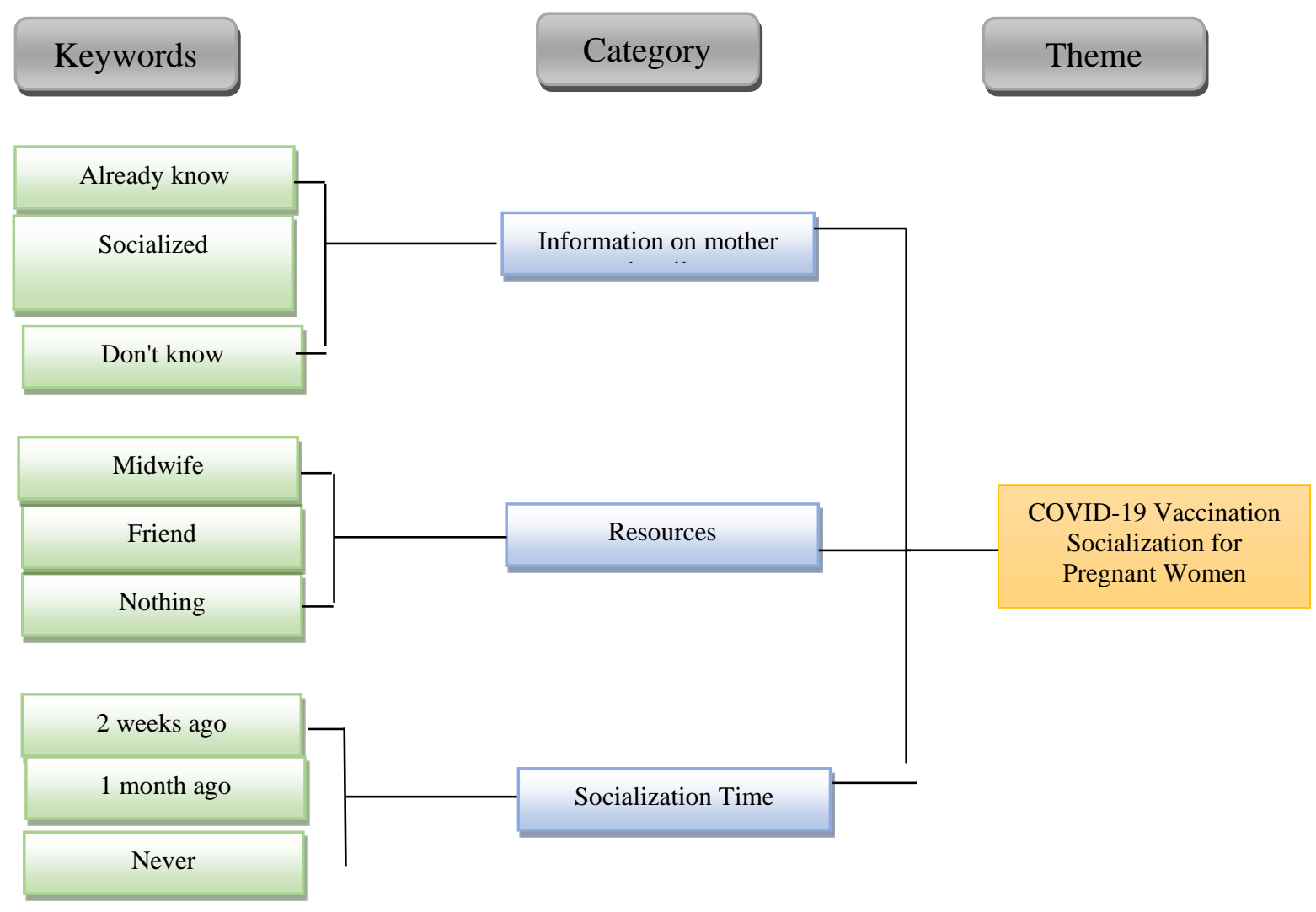

Figure 1. Theme I: Socialization of Covid-19 Vaccination for Pregnant Women

The theme of the socialization of Covid-19 vaccination for pregnant women was obtained from 3 categories, namely information on pregnant women, sources of information, and time of socialization. Information obtained from pregnant women related to the covid-19 vaccination program is considered new and from 7 informants there are 4 people who say they have received information as shown in the statement below:

“...Has anyone ever told you about it" (P1)

"Yes, I already know that I have heard of the vaccine for pregnant women" (P2, P5, P6)

"I don't know, because I've never heard of it" (P3)

"I don't know, there is no socialization"'” (P4, P7)

Sources of information obtained by participants related to Covid-19 vaccination services for pregnant women were obtained through midwives, friends, and others who said they did not know the source of the information. As stated by the participants below:

"Has anyone ever told you about it" (P1)

"Then the midwife explained" (P5)

"my friend told me" (P2)

"the female midwife who informed" (P6)

"Never received information that vaccines were for pregnant women" (P4, P7, P3)

Participants revealed when they received information related to covid-19 vaccination for pregnant women as follows: :

“At Posyandu yesterday, yes, 1 month ago, that means" (P1) 
"2 weeks ago it was seen on TV" (P3)

"When the moon came, my friend told me" (P3)

"I have never received this information, later this will only go to the Community Health Center (P4).

Meanwhile, according to key informants, namely the Telaga PKM Coordinator midwife, said that the implementation of socialization related to Covid-19 vaccination for pregnant women has been socialized at every visit to pregnant women and this program is delivered in the form of counseling by midwives or doctors, the program has been implemented since 3 months ago. As stated by key informants as follows:

"The COVID-19 vaccination program for pregnant women has been promoted since 2 months ago, this is something new for pregnant women, which at first didn't need to be vaccinated, we have socialized this program to pregnant women at every visit. pregnant women who are carried out by doctors and midwives directly through counseling, but they can't give leaflets, they just tell people that" (P8)

"Yes, so I gave this socialization of the Covid-19 vaccination program to pregnant women at the posyandu, there was information that those who wanted the vaccine could ask for a letter of approval from their husbands or family, this socialization so how many months ago was there an explanation, so 2 months ago staw is there for every posyandu, the implementation just wants to let you know it's a poly leaflet and a video that I just want to design wants to make this so they can read and read at home" (P9)

From the results of in-depth interviews and direct observations, it was found on theme 1 , namely the socialization of Covid-19 Vaccination for Pregnant Women, this is illustrated in 3 categories, namely information on pregnant women, sources of information, and when to get information. From the results of the statement, it was found that the socialization of the Covid19 vaccination for pregnant women had been carried out since 2 months ago, this socialization was only given in the form of counseling through oral delivery without being accompanied by media such as videos, animations or leaflets. Socialization is carried out directly by doctors and midwives every time they meet at posyandu services (Nirwana et al., 2015).. According to participants from 7 participants, 3 of whom had heard about maternal services for Covid-19 vaccination for pregnant women, they received information about 2 weeks - 1 month ago, information was obtained through midwives and friends. The form of socialization of Covid19 vaccination for pregnant women, according to 4 other participants, said that they had never heard of a Covid-19 vaccination for pregnant women. They had never received socialization from the Puskesmas, nor heard news from friends, family or through the media.

The lack of maximum socialization depicted in theme 1 has caused information on Covid-19 vaccinations to be uneven among pregnant women, this is evidenced by 7 participants, only 3 knew about Covid-19 vaccination. One of the causes of socialization is not optimal, one of which is caused by the lack of active and uneven socialization from health workers, in addition to the less than optimal extension media not equipped with display screens for materials, videos, and leaflets, as well as the lack of socialization media related to Covid-19 vaccination for pregnant women. in electronic media and mass media so that the information that reaches pregnant women is not evenly distributed and there is 1 participant who has just visited the PKM whose gestational age has only reached 10 weeks. This causes the Covid-19 vaccination target for pregnant women to be very low, namely 20-30\% for Gorontalo Province, while vaccination coverage for PKM Telaga still reaches 5-10\%. 
Dissemination of the Covid-19 vaccination is the main task of health workers in conveying information to pregnant women at every posyandu visit or at maternal services so that the target for Covid-19 vaccination for pregnant women can be achieved 80\% - 100\%, in addition to support from the health office as available vaccines also take part in this program, as well as the participation of the community and pregnant women to avoid getting wrong information. This Covid-19 vaccination policy has been issued by the Government through the Ministry of Health, initially vaccination was given to all except pregnant women, but in August 2021 the government issued a new policy regulation related to the appeal for Covid-19 vaccination for pregnant women as stated in the regulation. issued by the Ministry of Health to protect pregnant women and fetuses from COVID-19 infection, the Ministry of Health of the Republic of Indonesia ensures that pregnant women are one of the targets for receiving COVID-19 vaccinations. Efforts to provide COVID-19 vaccination to pregnant women have also been recommended by the National Immunization Expert Advisory Committee (ITAGI). This policy is contained in Circular HK.02.01/I/2007/2021 concerning COVID-19 Vaccination for Pregnant Women and Screening Adjustments in the Implementation of COVID-19 Vaccination on August 2, 2021.

\section{Theme 2: KIPI (Following Events Post-Covid-19) Issue Vaccination Immunization}

The second theme obtained in this study is the Issue of KIPI (Following Events Post-Covid19) Covid-19 Vaccination in pregnant women. To explore this, the researcher asked: "What is the mother's response to the side effects of the Covid-19 vaccination?" and obtained keywords and categories to describe the theme as shown in Figure 5.2 below:

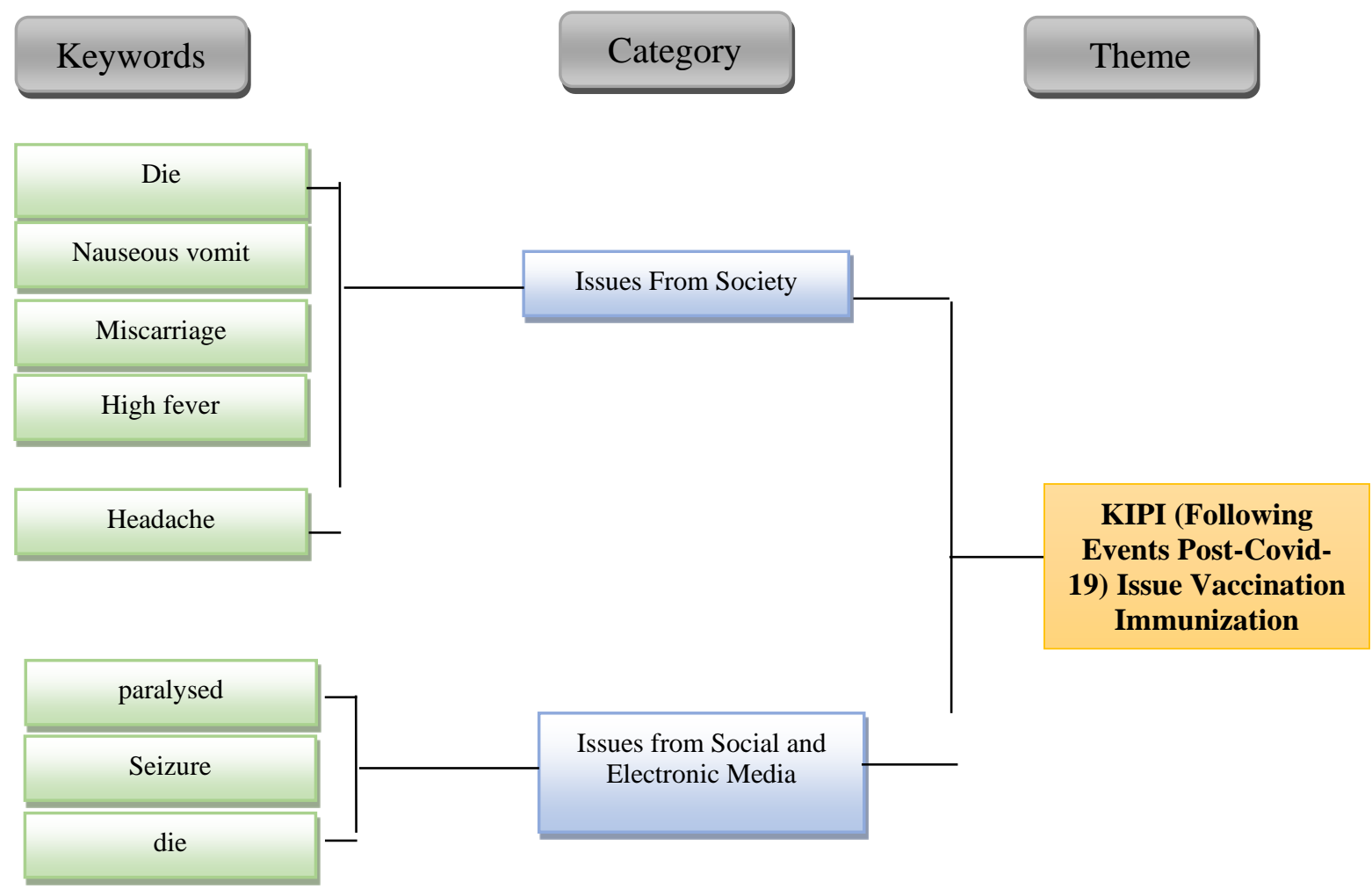

Figure 2. Theme 2: Covid-19 Vaccination KIPI Issue

Theme: AEFI Issue (After Immunization Follow-Up) Covid-19 vaccinations for pregnant women were obtained from 2 categories, namely issues from the community and issues from social and electronic media as seen in the participant statements below : 
"Corona has side effects, they say that many people die, have fever, some immediately have seizures, so I'm afraid because people say it's fine, I want to have a miscarriage after the vaccine" (P1, P6)

"Yes, there was a person near the house who finished the vaccine, he said he immediately felt a fever, then his head was really heavy, he said he felt like he was dizzy, he was vomiting again" (P4, P5)

"This is the same portal on TV, you can see people who have finished their vaccines, some people die, are paralyzed, have headaches, have high fever and chills" (P2, P5)

"It's on TV that people can see seizures immediately after the vaccine" (P3, P7)

Meanwhile, according to a key informant, namely the Coordinator Midwife of PKM Telaga, said that many issues spread in the community caused mothers to hesitate and even refuse to be vaccinated against COVID-19, the number of issues circulating in the community regarding the side effects of this vaccination, of course, caused concern for the community, especially for pregnant women because of this. is a new program and certainly has more risks to pregnant women in its implementation. The issue of KIPI events is of course very fast growing in the community, it is easy to get information from the media and events in the field that are informed to the public at large, one of which is the public assumes that many events have occurred after the vaccine, including high fever, seizures, dizziness, nausea and vomiting. until death

"The side effects or what people usually call KIPI are also many issues that are not true circulating in the community which of course makes the wider community afraid, especially pregnant women and their families. So far, both the Covid-19 vaccination for pregnant women and the general public, as well as the elderly. Until now, reports of KIPI incidence are around $2-4 \%$ and side effects that occur are only in mild categories such as fever, chills, and headaches.

"As for the side effects of vaccination, pregnant women only have fever in the arm, only mild fever, the rest is not available, for vaccines in the general public other than pregnant women, the average is almost the same, only fever, nausea, headache are the most but around 80-90\% there are no complaints felt by the community after the vaccine" (P9)

From the results of in-depth interviews and direct observations, it was found on theme 2, namely the AEFI issue on Covid-19 vaccination for pregnant women, this is illustrated in 2 categories, namely issues from the community and issues from social and electronic media. From the interviews, it was concluded that there were doubts, fears and unwillingness of pregnant women in implementing the vaccine, one of which was caused by the many issues circulating in the community related to side effects that occurred such as high fever, seizures, headaches, nausea, vomiting, paralysis, miscarriage and even die. This issue greatly affects the success of the implementation of the Covid-19 vaccination program for pregnant women because of the fear and worry if things happen that pose a risk to their pregnancy. The reality in the field is that the incidence of side effects or AEFIs is not the same as in issues that are spread in the community to cause death, this is usually accompanied by comorbidities and not caused by vaccines. The side effects that arise are only in the form of fever, nausea, headache, even $80-90 \%$ said they did not feel any side effects after being vaccinated against Covid-19, only mild pain around the injection in the arm.

The chairman of the National Commission for Post-Immunization Adverse Events (Komnas KIPI) Prof. Hindra Irawan Satari emphasized that so far no one has died from the COVID-19 vaccination. This needs to be re-explained considering the many confusing news relating to 
several cases of death due to the Covid Vaccine. According to Komnas KIPI, there were 27 cases of death suspected to have been caused by vaccination with Sinovac. However, upon investigation, the death was not related to vaccination. Of these cases, 10 were infected with Covid-19, then 14 were due to heart and blood vessel disease, 1 was due to sudden kidney function disorders and 2 were due to diabetes mellitus and uncontrolled hypertension. While those who died were thought to be due to vaccination with Aztra Zeneca, there were 3.

Post-Immunization Follow-up Events (AEFI) in COVID-19 vaccination are medical events that are suspected to be related to COVID-19 vaccination. Not everyone vaccinated against COVID-19 experiences a reaction or (AEFI). The emergence of a reaction or AEFI is something natural. AEFIs that appear after vaccination are much lighter than if they are exposed to COVID-19 or complications caused by the COVID-19 virus. Implementation of health protocols and vaccinations is the most appropriate way to get out of the COVID-19 pandemic. The appearance of AEFI after vaccination indicates that the vaccine is working in the body where the immune system is learning how to protect itself from disease. AEFIs are generally temporary and will go away on their own in a few days.

\section{Theme 3: Supporting Factors for Successful Covid-19 Vaccination for Pregnant Women}

Supporting Factors for the Success of Covid-19 Vaccination in pregnant women is one of the factors that can increase the goals and targets of this program. To explore in depth the supporting factors, the researcher asked the participants, namely: "What makes mothers want to carry out the Covid-19 vaccination?" and obtained keywords and categories to describe the theme as shown in Figure 3 below

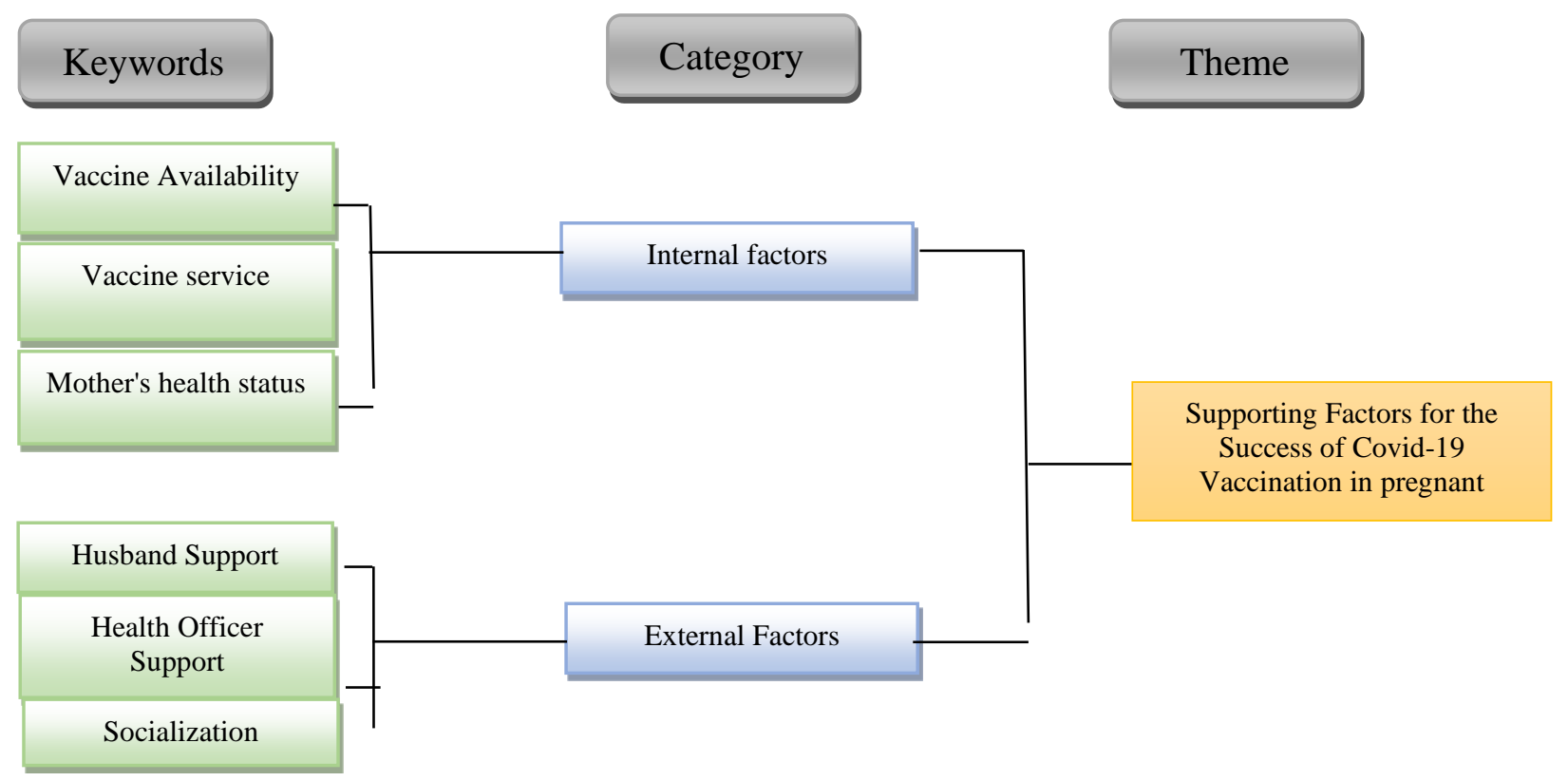

Figure 3. Theme 3: Supporting Factors for Successful Covid-19 Vaccination in Pregnant Women

Theme 3: Supporting Factors for the Success of Covid-19 Vaccination In pregnant women obtained from 2 categories, namely internal factors and external factors. Internal factors obtained are the availability of vaccines, vaccine services, and maternal health status, while external factors are obtained by husband's support, support from health workers and forms of socialization. As illustrated in the form of the following statement: 
"I've been vaccinated because (Health Officers) said this is a must if you are pregnant so you don't want to get sick, if you feel sick you can't get a vaccine or if you have a congenital disease you can't" (Pl)

"I depend if I have to vaccinate, if my husband allows me to vaccinate, well I vaccinate" $(P 2, P 5$, )

"Then it was because only a few wanted the vaccine, so it didn't happen because 4-5 people had to be vaccinated before getting vaccinated, so later I was quite ready to come" (P3)

"Hopefully this vaccine really doesn't want to get sick or have problems with children, I also have to break it first with my husband if my husband gives permission or not, the midwife should also want to tell this person that there has been no socialization" (P4 )

"I'm actually afraid of vaccines if I want to hear this news but if it's really good, which one is good if the midwife says it's good, then my husband will also give permission, God willing, he wants a vaccine" (P7, P6)

Meanwhile, according to a key informant, the Coordinator of PKM Telaga, there are many factors that support the success rate of Covid-19 vaccination in pregnant women, such as the availability of vaccines, the health status of the mother, the form of service, and support from husbands or families are also very important and of course socialization is also important. main key to success. As stated by the informant below:

"The success of the Covid-19 vaccination depends on the trust of pregnant women themselves so that socialization is very important, the support of husbands and health workers is a supporting factor for mothers to carry out vaccines, besides the availability of vaccines and forms of service also play an important role in supporting this program. If you want to do a vaccine, you must sign an informed consent or a consent form and must be accompanied by your husband and family" (P8)

"Whether or not pregnant women vaccinate usually when the mother gets good information, besides that support from family, health workers and husbands is very important, the health status of the mother is also because usually if the mother is sick or feels there is an accompanying disease, she doesn't want to be vaccinated even though In normal health status, vaccination can be carried out according to the results of screening and recommendations from doctors" (P9)

From the results of in-depth interviews and direct observations, it was found on theme 3, namely Supporting Factors for the Success of Covid-19 Vaccination. In pregnant women, this is illustrated by 2 categories, namely internal factors and external factors. From the results of the statement, it was found that socialization, vaccine availability and health status were internal factors for the success of Covid-19 vaccination for pregnant women, in addition to external factors with support from husbands, families and health workers, of course, were factors that could support the success of Covid-19 vaccination. in pregnant women. The many considerations that are felt by pregnant women become a form that changes the thinking of pregnant women to be able to vaccinate, with the support of course pregnant women feel calm in doing the vaccine because of the many supporting sources that are obtained from all parties although there are many negative issues from the community and the media. Sources of support are needed by pregnant women to provide calm, reduce stressors, and reduce the risks that can occur in their pregnancy.

With the issuance of the Covid-19 vaccination rules for pregnant women, the Ministry of Health has instructed all Heads of Provincial Health Offices, Heads of District/City Health 
Offices, and heads of health service facilities that carry out COVID-19 vaccinations, to immediately start vaccinating pregnant women, especially in areas with a high rate of transmission of COVID-19 cases. So an important role in the socialization and support of health workers as well as the availability of vaccines and services is an important role to support this program.

The regulation also explains that vaccination for pregnant women is included in special criteria. Therefore, the process of skinning/screening of the health status of the target before vaccination is carried out in more detail than other targets. The screening format on the control card for pregnant women has also been prepared by the Ministry of Health. The first dose of COVID19 vaccine will be given in the second trimester of pregnancy, and the second dose will be given according to the interval of the type of vaccine.

\section{Theme 4: The Response of the Body of Pregnant Women after the Covid-19 Vaccination}

The response of the pregnant mother's body after giving the Covid-19 vaccination to pregnant women is one of the supporting factors for this program. To delve deeply into the response of the body of pregnant women after the Covid-19 vaccination, the researcher asked 3 participants who had been vaccinated, namely: "How did the mother feel after being vaccinated?" and obtained keywords and categories to describe the theme as shown in Figure 4 below:

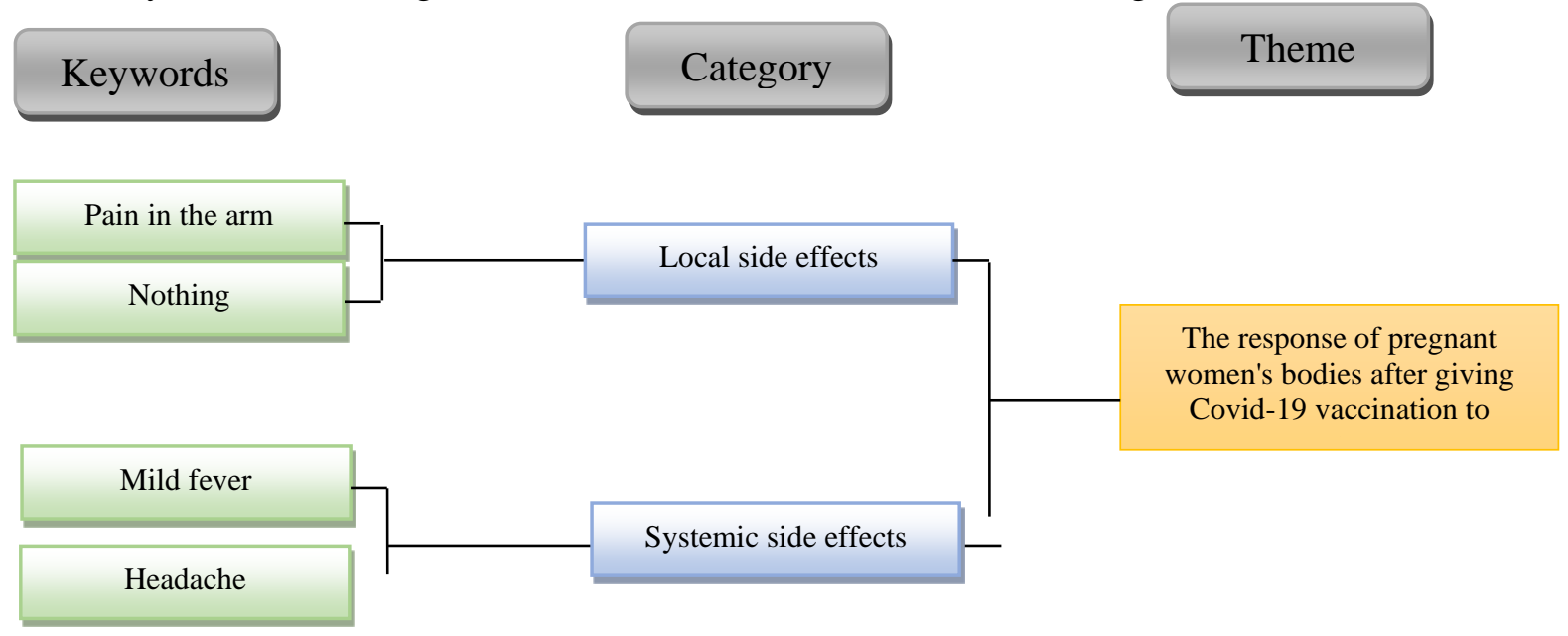

Figure 4. Theme 4: The response of the pregnant mother's body after giving the covid-19 vaccination

The theme of the response of pregnant women's bodies after being vaccinated was asked to 3 participants who had received the Covid-19 vaccine, namely participants 1, 2, and 5 and . As illustrated in the form of the following statement:

"Yesterday, the vaccine was finished. Thank God no one was sick, there were no complaints" (P1, P2)

"I finished the vaccine and then I got pain in my hand where I had an injection, but it took 2 days, so it's gone, Alhamdulillah" (P5)

Meanwhile, according to the key informant, namely the Telaga PKM Coordinator midwife, said that after the vaccine, the complaints of pregnant women were still limited to mild symptoms such as pain in the arm at the injection site, headache, and fever, but mostly there was no excessive body response or no complaints. As stated by the informant below: 
"So far, the complaints of mothers after the ba vaccine, Alhamdulillah, nothing is so severe, only about 1 in 20 people who are vaccinated complain of mild symptoms such as pain in the arm after the injection, fever, and headache" (P8)

"After the vaccine, Alhamdulillah, it wasn't that bad, mostly there were no complaints and then people complained about headaches, but this mother did have a history of hypertension, only when there was the DPE vaccine, the blood pressure was within normal limits. dpe, the house was flooded, it was rained, they said that it caused a fever," (P9)

From the results of in-depth interviews and direct observations, it was found on theme 4, namely the response of the body of pregnant women after giving the covid-19 vaccination, this is illustrated by the results of interviews by 3 participants and 2 key informants. From the results of the statement, it was found that in general the body's response that was caused after the pregnant woman was vaccinated was within normal limits as an antibody reaction to the virus that caused symptoms such as fever, pain, headache, but most of the mothers who had been vaccinated did not feel any complaints after the vaccination.

According to the Indonesian Obstetrics and Gynecology Association (POG) said that the symptoms caused by this vaccine were mild symptoms such as fever marked with body temperature above 37.80 , headache or muscle pain, nausea or vomiting, joint pain, chills, arm pain. at the injection site, fatigue, flu-like symptoms, chills for 1-2 days. Things to do if an AEFI occurs after COVID-19 vaccination is to get enough rest, drink enough water, if necessary take fever-reducing medication according to the recommended dose, if there is pain at the injection site, keep moving and use your arm as usual. Compress the painful area using a clean cloth soaked in cold water. If the fever appears more than 48 hours after vaccination or lasts longer than 48 hours, self-isolate and get tested for COVID-19, if the complaint does not subside, stay calm and contact the health worker at the contact number listed on the vaccination card.

Until now, there is no scientific data regarding the effectiveness or potential dangers of administering the Covid-19 vaccine for pregnant and lactating women, considering that it belongs to the vulnerable population group," Ari explained. In general in Indonesia, the 1 month interim safety data of the CoronaVac vaccine shows a comparable side effect profile between the vaccine group with placebo and generally mild to moderate. However, POGI acknowledged that there are some side effects of the Covid-19 vaccine in pregnant women, namely as follows: following: Pain, Redness, Swelling Meanwhile, the reported systemic side effects are as follows: Myalgia (Muscle pain), Fatigue (Excessive fatigue), and fever.

\section{Theme 5: Conditions for Giving Covid-19 Vaccination to Pregnant Women}

Conditions for giving Covid-19 vaccination to pregnant women. To delve deeply into the response of pregnant women's bodies after the Covid-19 vaccination, the researcher asked 4 participants who had been vaccinated, namely: "What do you know about the requirements for the covid-19 vaccination?" and obtained keywords and categories to describe the theme as shown in Figure 5 below: 


\section{Keywords}

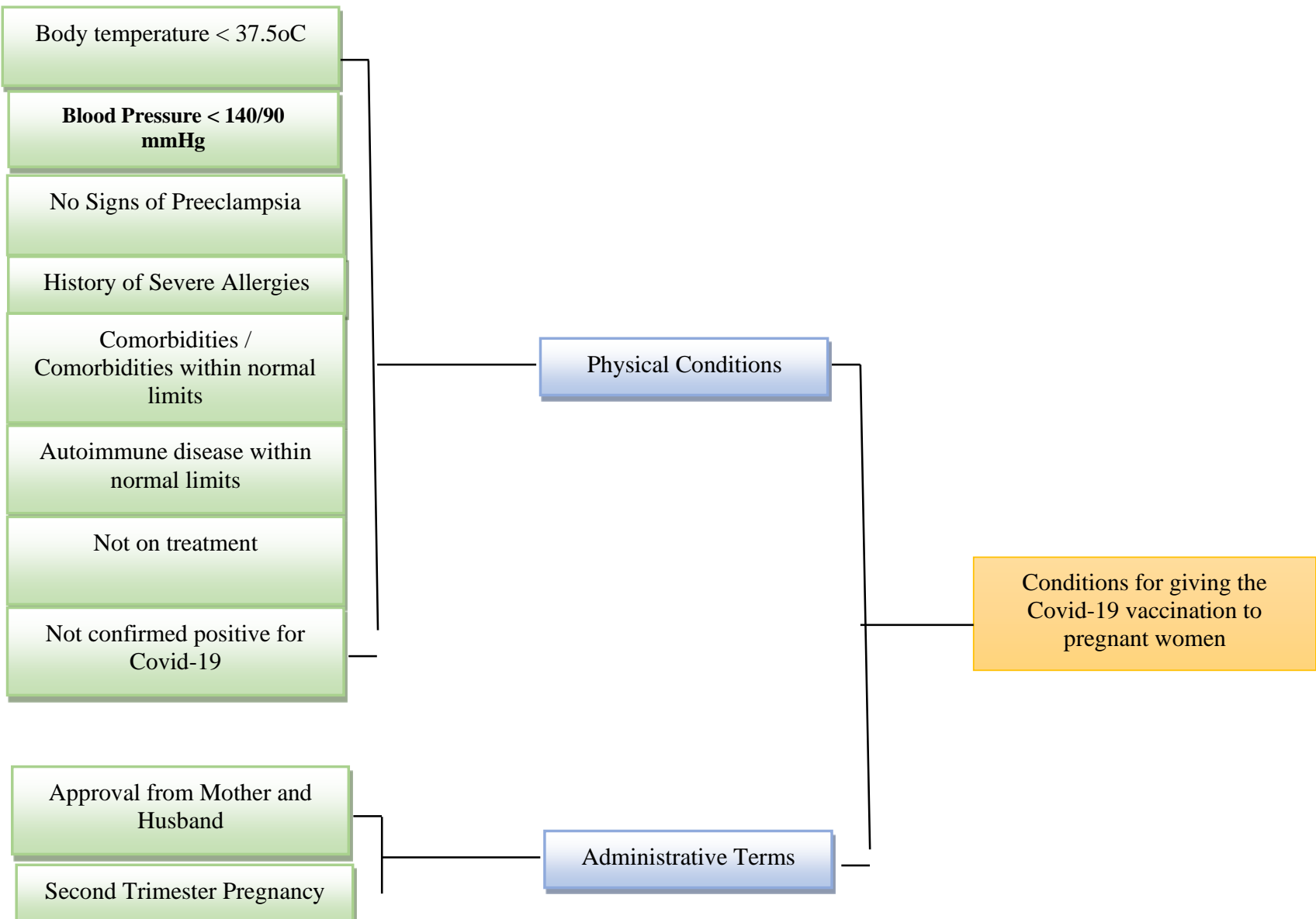

Figure 5. Theme 5: Conditions for Giving Covid-19 Vaccination to pregnant women

Theme 5: Requirements for Vaccination against Covid-19 for pregnant women revealed by 4 participants and $2 \mathrm{key}$ informants that the conditions for a pregnant woman to be vaccinated include body temperature $<37 \mathrm{oC}$, blood pressure $<140 / 90 \mathrm{mmHg}$, no preeclampsia, history of severe allergies , comorbidities or comorbidities within normal limits, autoimmune diseases within normal limits, not currently undergoing treatment, not confirmed positive for Covid-19. As illustrated in the form of the following statement:

"For the condition that the gestational age is above 4 months, then you are not sick, such as fever and blood pressure, you must have a letter of approval from your husband or family before you can get the vaccine" $(P 1, P 2)$

"Yesterday, the doctor said that the important thing is not to have shortness of breath, not to be allergic, not to be dizzy, or to have no congenital disease" (P5, P6)

Meanwhile, according to key informants, namely the Telaga PKM Coordinator midwife, said that the vaccine requirements for pregnant women were in accordance with the technical guidelines and through screening and family or husband approval, including body temperature $<37 \mathrm{oC}$, blood pressure $<140 / 90 \mathrm{mmHg}$, no preeclampsia, history of severe allergies, comorbidities or comorbidities within normal limits, autoimmune diseases within normal limits, not currently undergoing treatment, not confirmed positive for Covid-19. As stated by the informant below: 
"If in accordance with the technical guidelines, the requirements are through screening and family or husband approval, including body temperature $<37 o C$, blood pressure < 140/90 mmHg, no preeclampsia, history of severe allergies, comorbidities or comorbidities within normal limits, autoimmune diseases within normal limits. normal, not undergoing treatment, not confirmed positive for Covid-19 then the pregnancy in the second trimester just wants to give dose 1 vaccine" (P8)

"The requirements are in accordance with the protocol, you want to be screened first, given later in the second trimester of pregnancy, if the requirements are usually there must be a family or husband's approval sheet, including body temperature < $37 o C$, blood pressure $<140 / 90 \mathrm{mmHg}$, no preeclampsia, history of severe allergies, comorbidities or comorbidities within normal limits, autoimmune diseases within normal limits, not currently on medication, not confirmed positive for Covid-19," (P9)

From the results of in-depth interviews and direct observations, it was found on theme 5, namely the requirements for the provision of Covid-19 vaccination, this is illustrated by the results of interviews by 4 participants and 2 key informants. From the results of the statement, it was found that the vaccine requirements for pregnant women were in accordance with the technical guidelines and through screening and approval from the family or husband, including body temperature $<37 \mathrm{oC}$, blood pressure $<140 / 90 \mathrm{mmHg}$, no preeclampsia, history of severe allergies, comorbidities or comorbidities within normal limits. normal, autoimmune disease within normal limits, not on medication, not confirmed positive for Covid-19

The Ministry of Health, the Indonesian Technical Advisory Group on Immunization (ITAGI) or the National Immunization Expert, and the Indonesian Obstetrics and Gynecology Association (POGI) recommend giving vaccines to pregnant women. Before being allowed to receive the Covid-19 vaccine, pregnant women need to undergo a screening or screening process to see their health status. Based on Circular Letter HK.02.01/I/2007/2021 concerning Covid-19 Vaccination for Pregnant Women and Screening Adjustments in the Implementation of Covid-19 Vaccinations, the conditions for pregnant women to be vaccinated against Covid-19 include: Body temperature below 37.5 degrees Celsius Blood pressure below $140 / 90 \mathrm{mmHg}$. If the result is above $140 / 90 \mathrm{mmHg}$, the measurement is repeated 5-10 minutes later, if it is still above the threshold, the Covid-19 vaccination is postponed. Gestational age in the second trimester, or above 13 weeks No signs of preeclampsia such as legs swelling, headache, heartburn, blurred vision, and blood pressure above 140/90 mmHg No history of severe allergies such as shortness of breath, swelling, or hives all over the body For pregnant women with comorbidities such as heart disease, diabetes, asthma, lung disease, HIV, hyperthyroid/hypothyroid, chronic kidney disease, or liver disease; comorbidities under controlled conditions and no acute complications, for pregnant women with autoimmune diseases or undergoing autoimmune treatment such as lupus, disease under controlled conditions and no acute complications Not currently undergoing treatment for blood clotting disorders, blood disorders, immune deficiencies, and recipients product or blood transfusion Not currently receiving immunosuppressant treatment such as corticosteroids and chemotherapy Not confirmed positive for Covid-19 within the last three months For pregnant women.

\section{Conclusion}

After conducting research through in-depth interviews and direct observation, 5 themes were obtained that represent the Covid-19 vaccination service for pregnant women, namely; (1) Theme 1: Socialization of Covid-19 Vaccination, socialization that has not been evenly distributed to all pregnant women has led to a lack of participation of pregnant women to participate in Covid-19 vaccination services for pregnant women; (2) Theme 2: Issue of AEFI

Copyright @ 2021, Journal of Asian Multicultural Research for Medical and Health Science Study, Under the license CC BY-SA 4.0 
vaccination for Covid-19, issues related to the side effects of this vaccination which are widely circulated in the community so that it creates concern and fear for most pregnant women in vaccinating; (3) Theme 3: Supporting factors for the success of Covid-19 vaccination in pregnant women, supporting factors are certainly positive things for pregnant women who can increase their confidence to be able to carry out vaccinations such as support from family, husbands, health workers, and of course information which is clear through socialization or education; (4) Theme 4: Body Response After Being Given Covid-19 Vaccination In pregnant women, the body's response felt by 3 participants who had the vaccine in the form of mild symptoms such as pain in the arm where the injection was given and the rest had no complaints; (5) Theme 5: Conditions for Covid-19 vaccination for pregnant women, vaccination requirements for pregnant women of course through screening, approval from the husband and family, gestational age in the second trimester, and body condition and comorbidities within normal limits.

\section{References}

Alhomaizi, A., Alhomaizi, D., Willis, S., \& Verdeli, H. (2021). Social Distancing in the Era of COVID-19: A Call for Maintaining Social Support for the Maternal Population. Global Health: Science and Practice.

Blakeway, H., Prasad, S., Kalafat, E., Heath, P. T., Ladhani, S. N., Le Doare, K., ... \& Khalil, A. (2021). COVID-19 vaccination during pregnancy: coverage and safety. American Journal of Obstetrics and Gynecology.

Bookstein Peretz, S., Regev, N., Novick, L., Nachshol, M., Goffer, E., Ben-David, A., ... \& Yinon, Y. (2021). Short-term outcome of pregnant women vaccinated with BNT162b2 mRNA COVID-19 vaccine. Ultrasound in Obstetrics \& Gynecology, 58(3), 450-456.

Gupta, P. D., \& Pushkala, K. (2021). How COVID-19 Affects Expecting Women?. Immunity, 12, 13.

Hanson, M., Barker, M., Dodd, J. M., Kumanyika, S., Norris, S., Steegers, E., ... \& Yang, H. (2017). Interventions to prevent maternal obesity before conception, during pregnancy, and post partum. The lancet Diabetes \& endocrinology, 5(1), 65-76.

Kerber, K. J., de Graft-Johnson, J. E., Bhutta, Z. A., Okong, P., Starrs, A., \& Lawn, J. E. (2007). Continuum of care for maternal, newborn, and child health: from slogan to service delivery. The Lancet, 370(9595), 1358-1369.

Nirwana, M. D., Utami, I. H., \& Utami, H. N. (2015). The Cadre of Integrated Health Service Post (Posyandu) as an Agent in the Socialization of Cervical Cancer Prevention in Malang Regency, Indonesia: A Cultural Approach. Procedia-Social and Behavioral Sciences, 211, 681-687.

Steen, M., Downe, S., Bamford, N., \& Edozien, L. (2012). Not-patient and not-visitor: a metasynthesis fathers' encounters with pregnancy, birth and maternity care. Midwifery, 28(4), 422-431.

Sulkowski, L., \& Ignatowski, G. (2020). Impact of COVID-19 pandemic on organization of religious behaviour in different Christian denominations in Poland. Religions, 11(5), 254. 